\title{
Drug Discovery and Development: An Insight into Pharmacovigilance
}

\author{
Prashansa Agrawal ${ }^{*}$ \\ College of Pharmacy, The University of Arizona, Tucson, Arizona, USA \\ *Corresponding author: Prashansa Agrawal, College of Pharmacy, The University of Arizona, Tucson, Arizona, 85721, USA. Tel: +1 520- 626-6749, E-mail: \\ agrawal@pharmacy.arizona.edu
}

Received date: June 16, 2014, Accepted date: June 19, 2014, Published date: June 27, 2014

Copyright: () 2014 Agrawal P. This is an open-access article distributed under the terms of the Creative Commons Attribution License, which permits unrestricted use, distribution, and reproduction in any medium, provided the original author and source are credited.

\section{Editorial}

Drug discovery and development is a long-term, competitive, expensive and complicated process. Bringing the drugs from the bench to the market, that is, from screening and identification of the drug to its introduction to the market, takes several years of efforts [1]. The process of discovering and developing a new drug involves an intricate interaction between investors, industry, academia, patent laws, regulatory authorities, marketing and the necessity to balance confidentiality with communication [2]. The complete process of presenting a drug to the patients involves four stages- drug discovery, drug development, regulatory review and approval, and marketing [3].

\section{Drug Discovery}

In the drug discovery process, the first step is to identify an appropriate 'druggable' target [4], which can be a biomolecule or a protein receptor that is explicitly associated with a disease condition or pathology. After the target has been identified, the next step involves target validation and the confirmation of its role in the disease progression. This is followed by testing of the target against different small molecule compounds to identify lead compounds which can interact with the target biomolecule and display the potential to either nullify or slow the disease development. The lead compounds can be identified by screening a library of compounds through various methods, such as high-throughput screening [5], de novo synthesis [6], and isolation from the natural products [7].

\section{Drug Development}

The drug development phase involves stringent testing and optimization of the selected compounds to identify the 'drug candidate' which might be most effective in terms of safety, toxicity [8], dosage, and efficacy. For this purpose, the selected lead compounds are tested in cells (in vitro) and in animals (in vivo) to study their pharmacodynamic and pharmacokinetic properties, which include Absorption, Distribution, Metabolism, Excretion and Toxicity (ADME/Tox) properties. The successful lead candidate must be nontoxic and should be absorbed into the bloodstream, can be distributed to the proper action site in the body, and can be metabolized efficiently and effectively as well as successfully excreted from the body. This part of the development process is referred to as the 'preclinical phase' in which the drug candidate is meticulously examined, optimized, and prepared for testing in humans. This phase is followed by the 'clinical phase' of development, in which the efficacy and safety of a drug candidate is scrutinized in patients. This 'clinical trial' has 3 phases: Phase 1 perform initial human testing in a small group of healthy volunteers, phase 2 involves testing in a small group of patients and phase 3 includes testing a large group of patients to show safety and efficacy of the drug candidate in them since the healthy and sick people have potentially different metabolic patterns for the drugs.

\section{Regulatory Review and Approval}

The outcome of the 'clinical trial' decides whether the drug candidate is safer and effective enough in treating the disease. At this point, new drug applications with all the essential evidence, including quality, preclinical and clinical data collected during development of the drug candidate, are submitted to the relevant regulatory authorities, e.g., the United States Food and Drug Administration (USFDA), which oversees the development, approval, and marketing of drugs [9]. They need to approve the drug applications so that the company can commercialize the drug in their jurisdictions (e.g., a New Drug Application (NDA) in USA, and Marketing Authorization Application (MAA) in Europe).

\section{Commercialization}

This is the last phase of drug development process. Once the drug has been approved, it is marketed or commercialized. The drug manufacturers need to submit marketing authorization applications in every country in which they want to sell the drug. Following this, postmarketing surveillance [10] are conducted by the manufacturer to continue evaluating the safety and efficacy of the marketed drug, and to better design its further development. These studies are considered as phase 4 clinical trials and are compulsory in some countries, e.g, Japan and Philippines. As the drug is typically targeted to a very large number of patients, the manufacturer is expected to monitor this stage cautiously and submit reports to the FDA. The reports include evidence for medicine-related problems, e.g., treatment failure, adverse reaction, counterfeit/poor quality medicines, drug interactions, or incorrect use. These reports are significant in terms of generating proof of efficacy that will inspire public confidence and trust.

The entire process of drug discovery and development costs around $\$ 0.8-1.0$ billion per drug [11,12] and takes about $10-15$ years and even then, there is a very high attrition rate for new drug candidates in clinical stage. To deal with the issues involved in failure of new drugs, USFDA has launched the "Critical Path Initiative and the Critical Path Opportunity" program in 2004 to guide the development process of new drug [13].

Introducing a new medicine to the patients is not only a highly time-consuming and expensive process but also requires an extremely essential and strict vigilance on the safety and efficacy of the drug. Therefore pharmacovigilance plays a pivotal role in drug discovery and development. The drugs may appear to be safe and well-tolerated in preclinical and clinical testing, but their safety in the 'real world' may not be distinct, after-effects of the drug when used frequently or in combination with other drugs are generally unknown, safety in 
Citation: Agrawal P (2014) Drug Discovery and Development: An Insight into Pharmacovigilance . J Pharmacovigilance 2: e120. doi:

Page 2 of 2

vulnerable groups with different metabolic profiles (e.g, pregnant women and breastfeeding mother, elderly person, young children) can be uncertain, and rumors and myths can destroy the integrity, adherence to, and success of a treatment.

Pharmacovigilance is the pharmacological science which deals with drug safety including accumulation, detection, assessment, monitoring, and prevention of adverse effects of the drugs. It is an iterative process focusing on detection of unidentified safety issues, identification of risk factors, quantifying risks and preventing patients from being adversely affected unnecessarily. Pharmacovigilance plays a critical role at various stages of drug discovery and development process; for example, in clinical research, pharmacovigilance requires submission of the reports on adverse events during clinical trials to regulatory authorities within a specified time frame, notification of such events to all investigators and ethics committees, and a safety review by independent Drug Safety Monitoring Boards (DSMB). Annual reports, a summary and analysis of all the serious adverse events, new safety findings from animal studies, and evaluations of benefit and risk are also required. Pharmacovigilance also plays a significant role when the drug is commercialized. Reporting the safety reviews is mandatory for companies in a marketing phase. These safety reviews include Risk Management Plan (RMP), Periodic Benefit Risk Evaluation Report (PBRER), the Development Safety Update Report (DSUR) [14], Periodic Safety Updates Report (PSUR) [15], phase 4 studies (post-marketing surveillance), clinical trials (intervene disease management), and pharmacoepidemiological studies (noninterventional or observational).

Pharmacovigilance is a very critical and inevitable part of the drug discovery and development process. It will require comprehensive documentation and severe monitoring at every phase of drug development including pharmacovigilance inspection and audit, risk management, and reporting of ADR medicinal drugs, periodic safety update report, post-authorization safety studies, additional monitoring, and safety communication. Therefore, it is absolutely essential to establish good pharmacovigilance practices for improving the understanding of the drug safety issues during the drug development and its post-approval so that the attrition rates can be reduced and the patients can be provided with safe and efficacious innovative medicines to meet their prerequisite medical needs.

\section{References}

1. Dimasi JA (2001) New Drug Development in the United States from 1963-1999. Clin Pharmacol Ther 69: 286-296.

2. Warren JB (2011) Drug discovery: lessons from evolution. Brit J Clin Pharmaco 71: 497-503.

3. http://cisncancer.org/

4. Rask-Andersen M, Almén MS, Schiöth HB (2011) Trends in the exploitation of novel drug targets. Nat Rev Drug Discov 8: 549-590.

5. Zhang XHD (2011) Optimal High-Throughput Screening: Practical Experimental Design and Data Analysis for Genome-scale RNAi Research. Cambridge University Press, New York.

6. Schneider G, Fechner U (2005) Computer-based de novo design of druglike molecules. Nat Rev Drug Discov 4: 649-663.

7. Newman DJ, Cragg GM (2007) Natural products as sources of new drugs over the last 25 years. J Nat Prod 70: 461-477.

8. Robinson S, Delongeas JL, Donald E, Dreher D, Festag M, et al. (2008) A European pharmaceutical company initiative challenging the regulatory requirement for acute toxicity studies in pharmaceutical drug development. Regul Toxicol Pharmacol 50: 345-352.

9. Meadows M (2002) The FDA's Drug Review Process: Ensuring Drugs are Safe and Effective. FDA Consum 36: 19-24.

10. McNeil JJ, Piccenna L, Ronaldson K, Ioannides-Demos LL (2010) The Value of Patient-Centred Registries in Phase IV Drug Surveillance. Pharm Med 24: 281-288.

11. DiMasi JA, Hansen RW, Grabowski HG (2003) The Price of Innovation: New Estimates of Drug Development Costs. J Health Econ 22: 151-185.

12. Adams CP, Brantner VV (2010) Spending on new drug development. Health Economics 19: 130-141.

13. U.S. Department of Health and Human Services (2004) Innovation or Stagnation: Innovation or Stagnation? Challenge and Opportunity on the Critical Path to New Medical Products. Food and Drug Administration.

14. Department of Health and Human Services (2003) Safety Reporting Requirements for Human Drug and Biological Products, Food and Drug Administration.

15. Klepper MJ (2004) The periodic safety update report as a pharmacovigilance tool. Drug Safety 27: 569-578. 